\title{
Effects of N-terminus modifications on the conformation and permeation activities of the synthetic peptide L1A
}

\author{
Luciana Puia Moro Zanin ${ }^{1}$ - Alexandre Suman de Araujo ${ }^{1}$ () • \\ Maria Aparecida Juliano ${ }^{2} \cdot$ Tiago Casella $^{3} \cdot$ Mara Correa Lelles Nogueira ${ }^{3}$. \\ João Ruggiero Neto ${ }^{1}$
}

Received: 24 December 2015 / Accepted: 6 February 2016 / Published online: 27 February 2016

(C) Springer-Verlag Wien 2016

\begin{abstract}
We investigate the effect of the N-terminus modification of the L1A, a synthetic octadecapeptide, on its helical content, affinity and lytic action in model membranes and on its hemolytic and antibacterial activities. L1A and its acetylated analog displayed a selective antibacterial activity to Gram-negative bacteria without being hemolytic. The covalently linked 2 -aminobezoic acid to the $\mathrm{N}$-terminus impaired the antibacterial efficacy and increased hemolysis. Despite their lower net charge $(+2), \mathrm{N}$-terminus modifications resulted in enhanced affinity and improved lytic efficiency in anionic vesicles. The analogs also showed higher helical content and consequently higher amphipathicity in these vesicles. The conformational analysis by molecular dynamics simulations in $30 \%$ of TFE/water showed that the hydrophobic faces of the peptides are in close contact with CF3 groups of TFE while the hydrophilic faces with water molecules. Due to the loss of the amino charge, the N-termini of the analogs are buried in TFE molecules. The analysis of the pair distribution functions, obtained for the center of mass of the charged
\end{abstract}

Handling Editor: J. D. Wade.

Alexandre Suman de Araujo

asaraujo@ibilce.unesp.br

João Ruggiero Neto

jruggiero@sjrp.unesp.br

1 Department of Physics, IBILCE, São Paulo State University, Rua Cristovão Colombo 2265, São José do Rio Preto, SP CEP 15054-000, Brazil

2 Department of Byophysics, UNIFESP, São Paulo, Brazil

3 Department of Dermatological, Infectious and Parasitary Diseases, FAMERP, São Jose do Rio Preto, SP, Brazil groups, has evidenced that the state of the $\mathrm{N}$-terminus has influenced the possibility of different ion-pairing. The higher complexity of the bacterial cells compared with anionic vesicles hampers to establish correlations structure-function for the analogs.

Keywords Antimicrobial peptide · Peptide selectivity · Lytic activity $\cdot$ Biological activity $\cdot$ Molecular dynamics

\section{Introduction}

Helical cationic peptides constitute an important class of lytic and antimicrobial peptides, which are broadly distributed among many living species and belong to the innate immune systems of these species (Zasloff 2002; Hamill et al. 2008). Generally, antimicrobial peptides are composed of 10-50 amino acids that are rich in positive charges and hydrophobic residues which are distributed in such way that they form an amphipathic helical structure when in contact with lipid bilayer or membrane mimetic environment (Zasloff 2002). Several studies (Teixeira et al. 2012; Pushpanathan et al. 2013; Souza et al. 2015) have indicated that formation of an amphipathic and stable structure on the lipid bilayer-solvent interface is capable to disturb the lipid packing of the bilayer giving rise a lytic process in bacterial membranes, responsible for the peptides antimicrobial activity.

We have observed that the presence of acidic residues near the N-terminus contribute in stabilizing the amphipathic helical structure of MP1. Although acidic residues are rarely found in antimicrobial peptides (AMP) (Wang and Wang 2004; Fjell et al. 2007), in the case of MP1 it seems that they play key role in modulating its selectivity. The substitution of the aspartic residue in the $\mathrm{N}$-terminus 
by an asparagine (D2N) increased significantly the hemolytic activity (Leite et al. 2011). In addition, the substitution of the aspartic residues by lysines and the glutamine by arginine in the MP1 resulted in higher toxicity to human cells (Wang et al. 2010). Molecular dynamics (MD) simulation in $30 \%$ TFE (dos Santos Cabrera et al. 2008) suggested an important role played by the acidic residue in the $\mathrm{N}$-terminus as well as the relative positioning of acidic and basic residues on the charge-hydrophobic balance.

The rationale behind the helical stabilization of the helical structure due to the acidic residue near the $\mathrm{N}$-terminus is that this residue decreases the repulsion between the amino group and the helix macrodipole (dos Santos Cabrera et al. 2008). Similar stabilization effect was observed by chemical modification of N-terminus (Fairman et al. 1989). These modifications will also block the $\mathrm{N}$-terminus, preventing the peptide of proteolytic digestion that has been shown to be an alternative resistance mechanism for some strains (Andreu and Rivas 1998).

The main purpose of the present work was to investigate if the $\mathrm{N}$-terminal modifications stabilize the helical conformation of the antimicrobial peptide L1A (IDGLKAIWKKVADLLKNT-NH2) and if these modifications influence the lytic activity in liposomes and their biological activities. In addition, by conformational analysis by circular dichroism (CD) and MD simulations we looked to understand the mechanisms favoring this stabilization. We used two analogs of this peptide. One of them, AcL1A, the N-terminus was acetylated and the other, AbzL1A-W8V, the fluorescent moiety ortho-aminobenzoic acid (Abz) was covalently bonded to the $\mathrm{N}$-terminus and the tryptophan residue was substituted by a valine. These modifications result in a net charge $Q=+2 e$ in both cases. The N-terminus acetylation, associated with the $\mathrm{C}$-terminal amidation, are the classical modifications to prevent proteolytic digestion. Besides blocking the $\mathrm{N}$-terminus, the attachment of the fluorescence label was done for further applications of this analog in fluorescence microscopy experiments.

In this work the conformational analysis by $\mathrm{CD}$ and by MD simulations in association with their biophysical characterization in solution is presented with their antimicrobial and hemolytic evaluations. This investigation revealed that the N-terminus acetylation resulted in higher helical stability in TFE and in lipid vesicles compared to L1A. Probably due to the high anionic character of the bacteria outer membrane the reduction of the peptide net charge impaired the antibacterial activity in lesser extension to the acetylated analog compared with the unmodified peptide while both are non-hemolytic.

\section{Materials and methods}

Lipids: 1-palmitoyl-2-oleoyl-sn-glycero-3-phosphocholine (POPC) and 1-palmitoyl-2-oleoyl-sn-glycero3-phosphoglycerol (POPG) were purchased from Avanti Polar Lipids (Alabaster, AL, USA); carboxyfluorescein (CF), chloramphenicol and triphenyltetrazolium chloride (TCC) from Sigma Chemical Co (S. Louis, MI, USA). Tris(hydroxymethyl) aminomethane (Tris) were purchased from Merck. Unless otherwise indicated other chemicals were of high-quality analytical grade.

\section{Synthetic peptides}

The peptides IDGLKAIWKKVADLLKNT-NH2 (L1A), Ac- IDGLKAIWKKVADLLKNT-NH2 (Ac-L1A) and AbzIDGLKAIVKKVADLLKNT-NH2 (Abz-L1A-W8V) were obtained in an automated bench-top simultaneous multiple solid-phase peptide synthesizer (PSSM 8 system from Shimadzu) was used for the solid-phase synthesis of all the peptides by the Fmoc-procedure ( Korkmaz et al. 2008). The final peptides were deprotected in TFA and purified by semi-preparative HPLC using an Econosil C-18 column $(10 \mu, 22.5 \times 250 \mathrm{~mm})$ and a two-solvent system: (A) trifluoroacetic acid (TFA)/ $\mathrm{H}_{2} \mathrm{O}(1: 1000)$ and (B) TFA/acetonitrile $(\mathrm{ACN}) / \mathrm{H}_{2} \mathrm{O}$ (1:900:100). The column was eluted at a flow rate of $5 \mathrm{~mL} / \mathrm{min}$ with a 10 (or 30)-50 (or 60) \% gradient of solvent B over 30 or $45 \mathrm{~min}$. Analytical HPLC was performed using a binary HPLC system from Shimadzu with a SPD-10AV Shimadzu UV-vis detector, coupled to an Ultrasphere C-18 column $(5 \mu, 4.6 \times 150 \mathrm{~mm})$ which was eluted with solvent systems $\mathrm{A}_{1}\left(\mathrm{H}_{3} \mathrm{PO}_{4} / \mathrm{H}_{2} \mathrm{O}, 1: 1000\right)$ and $\mathrm{B}_{1}\left(\mathrm{ACN} / \mathrm{H}_{2} \mathrm{O} / \mathrm{H}_{3} \mathrm{PO}_{4}, 900: 100: 1\right)$ at a flow rate of $1.0 \mathrm{~mL} / \mathrm{min}$ and a $10-80 \%$ gradient of $B_{1}$ over $20 \mathrm{~min}$. The HPLC column eluates were monitored by their absorbance at $220 \mathrm{~nm}$. TFA desalination was done by multiple steps of lyophilization, in each step the product of previous step was solubilized in pure water and then frozen and lyophilized. The molecular weight and purity of synthesized peptides were checked by MALDI-TOF mass spectrometry (Bruker Daltons) or eletron spray LC/MS-2010 (Shimadzu).

\section{Peptide solution}

Stock solutions of peptides were prepared in Milli-Q water. Peptides were dissolved in TRIS buffer (tris/ $\mathrm{HCl} 10 \mathrm{mM}$, $1 \mathrm{mM} \mathrm{Na}{ }_{2}$ EDTA, $150 \mathrm{mM} \mathrm{NaCl}, \mathrm{pH} 7.5$ for Zeta experiments and dye leakage or tris $/ \mathrm{H}_{3} \mathrm{BO}_{3} 5 \mathrm{mM}, 1 \mathrm{mM} \mathrm{Na} \mathrm{E}_{2}$ DTA, $150 \mathrm{mM} \mathrm{NaF}$, pH 7.5 for CD experiments), under gentle agitation. Peptide concentration was determined spectrophotometrically with a Varian 2200 (Palo Alto, 
USA) spectrophotometer in $280 \mathrm{~nm}$ using molar extinction coefficient $\varepsilon=5675$ and $1998 \mathrm{M}^{-1}$.

\section{Antimicrobial activity}

The minimal inhibitory concentrations (MIC) of the peptides were determined based on methods described (Meletiadis et al. 2000). The following microorganisms' strains were used: Staphylococcus aureus (ATCC 25923), Escherichia coli (ATCC 35218) and Pseudomonas aeruginosa (ATCC 27853). The experiment was performed in 96 well plates. Bacterial cells were suspended in sterile culture medium; the inoculum size was $1 \times 10^{4}$ cells $\mathrm{m} / \mathrm{L}$ in Müller-Hinton broth (DIFCO), confirmed by the use of McFarland scale. From this culture, $50 \mu \mathrm{L}$ was spread onto the micro-plate previously containing $50 \mu \mathrm{L}$ of Müller-Hinton broth, resulting in a final cell density of the $2.5 \times 10^{3}$ cell/ $\mathrm{mL}$. Cells were incubated at $37{ }^{\circ} \mathrm{C}$ for $18 \mathrm{~h}$ in the presence of $100 \mu \mathrm{L}$ of each peptide solution, in a concentration range from 2.3 to $150 \mu \mathrm{g} / \mathrm{mL}$. After incubation, $10 \mu \mathrm{L}$ of a triphenyltetrazolium chloride (TTC) solution (final concentration $0.05 \%, w / v)$ was added to each well plate. The plate was incubated at $37^{\circ} \mathrm{C}$ for $2 \mathrm{~h}$. Alive colonies reduce TTC to a dark red color, while those with reduced respiratory function are unable to do this and it remains without color change. Thus, the results were expressed as the minimal concentration that inhibits all colony forming units. Chloramphenicol in a concentration ranging from 0.078 to $40 \mu \mathrm{g} / \mathrm{mL}$ was used as standard antibiotic and the negative controls were considered those wells containing only the culture medium. Results were expressed as the mean of three experiments.

\section{Hemolytic activity}

The hemolytic activities of peptides were assessed using human blood cell collected in vacuplast contain EDTA. $\mathrm{K} 3$ from healthy donors. The fresh blood were rinsed three times in PBS-1 $\left(10 \mathrm{mM} \mathrm{Na} 2 \mathrm{HPO}_{4} / \mathrm{KH}_{2} \mathrm{PO}_{4}, 136 \mathrm{mM} \mathrm{NaCl}\right.$, $3 \mathrm{mM} \mathrm{KCl}, \mathrm{pH} 7.4$ ), collected by centrifugation for $5 \mathrm{~min}$ at $845 \mathrm{xg}$ and re-suspended at $8 \%$ in PBS-2 $\left(50 \mathrm{mM} \mathrm{Na}_{2} \mathrm{HPO}_{4} /\right.$ $\mathrm{KH}_{2} \mathrm{PO}_{4}, 100 \mathrm{mM} \mathrm{NaCl}, 0.5 \mathrm{mM}$ EDTA, pH7.4) and incubated with sample for $60 \mathrm{~min}$ at $37^{\circ} \mathrm{C}$ with final concentration of the $300 \mu \mathrm{g}$. After incubation the samples then centrifuged for $5 \mathrm{~min}$ at $845 \times \mathrm{g}$. The absorbance of the supernatants was assessed at $540 \mathrm{~nm}$ and expressed as percentage of lysis. The controls for zero (blank) and $100 \%$ hemolysis were blood cell suspended in PBS-2 and $1 \%$ Triton X-100, respectively.

\section{Vesicle preparation}

Lipid films of POPC or mixture POPC/POPG at molar ratio (80:20) were obtained from lipids dissolved in chloroform or chloroform:metanol (2:1) in round-bottom flasks and the solvent was first dried under $\mathrm{N}_{2}$ flow and in vacuum overnight to remove traces of organic solvents. The lipid films were afterwards hydrated with TRIS buffer $\left(\right.$ Tris $/ \mathrm{H}_{3} \mathrm{BO}_{3}$ $5 \mathrm{mM}, 1 \mathrm{mM} \mathrm{Na}{ }_{2}$ EDTA, pH 7.5 or with Tris/HCl $10 \mathrm{mM}$, $1 \mathrm{mM} \mathrm{Na} 2$ EDTA, pH 7.5, either containing $25 \mathrm{mM}$ carboxyfluorescein $(\mathrm{CF})$ for leakage experiments or $150 \mathrm{mM}$ $\mathrm{NaCl}$ for fluorescence spectroscopy or $150 \mathrm{mM} \mathrm{NaF}$ for $\mathrm{CD}$ experiments. The final total lipid concentration was $5 \mathrm{mM}$. Small unilamelar vesicles (SUVs), for CD experiments, were obtained by sonication of the lipid suspension under $\mathrm{N}_{2}$ flow, in ice/water bath, for $50 \mathrm{~min}$, or until clear. Titanium debris has been removed by centrifugation. Large unilamellar vesicles (LUVs $\sim 5-10 \mathrm{mM}$ ) used in dye leakage experiments and zeta potential titrations were obtained by two extrusion steps using an Avanti Mini-Extruder (Alabaster, AL, USA) and double stacked polycarbonate membrane (Nuclepore Track-etch Membrane, Whatman): firstly 6 times through $0.4 \mu \mathrm{m}$ and then 11 times through $0.1 \mu \mathrm{m}$ membranes. For the dye-entrapped LUVs the free dye was separated by gel filtration on a Sephadex G25 M column (Amersham Pharmacia, Upsala, Sweeden). Vesicles were used within $24 \mathrm{~h}$ of preparation. The lipid concentration was determined by phosphorus analysis (Rouser et al. 1970). Laser light scattering measurements with Zeta Sizer Nano NS-90 (Malvern Instruments, Worcestershire, UK) have revealed homodisperse LUV suspensions, with an average diameter of $105 \pm 5 \mathrm{~nm}$ among several preparations.

\section{Lytic activity}

Aliquots of LUV suspension were added to $1 \mathrm{~cm}$ quartz cuvettes containing magnetically stirred peptide solutions to give a final volume of $1.2 \mathrm{~mL}$. Release of CF from LUVs causes decrease in its self-quenching that was followed with a ISS PC1 spectrofluorometer (Champaign, IL, USA) at $520 \mathrm{~nm}, 0.5 \mathrm{~nm}$ slit width, (excited at $490 \mathrm{~nm}, 1 \mathrm{~nm}$ slit width) at $25^{\circ} \mathrm{C}$, during $30 \mathrm{~min}$ for POPC/POPG (80:20) or POPC LUVs respectively. At regular intervals \% leakage is calculated as $100 \times\left(F-F_{0}\right) /\left(F_{100}-F_{0}\right)$, where $F$ is the measured fluorescence intensity, $F_{0}$ and $F_{100}$ correspond, respectively, to the fluorescence intensities in the absence of peptides and to $100 \%$ leakage, determined by the addition of $33 \mu \mathrm{L}$ of $6 \%$ Triton X-100 solution. $F_{100}$ has been corrected for the corresponding dilution factor. $F_{100} / F_{0}$ for this $\mathrm{CF}$ concentration is around 10 and 15 so we are assuming that in this $\mathrm{CF}$ concentration its fluorescence $F_{0}$ is completely self-quenched.

\section{Electrophoretic Mobility Measurements}

Electrophoretic mobility of anionic POPC:POPG (80:20) large unilamellar vesicles, at $40 \mu \mathrm{M}$ total lipid concentration, 
in the absence and in the presence of different concentrations of peptides, was determined from the electrophoretic mobility using a ZetaSizer Nano ZS90 (Malvern Instruments Ltd, Worcestershire, UK). Vesicles were prepared in tris buffer Tris/HCl 10 mM, 1 mM Na 2 EDTA, pH 7.5, $150 \mathrm{mM} \mathrm{NaCl}$, and measurements were carried out with a DTS1060 (Malvern) disposable cells with golden electrodes at $25{ }^{\circ} \mathrm{C}$. Typically the voltage applied in the conditions used is around $50 \mathrm{~V}$.

\section{Conformational analysis by circular dichroism (CD)}

CD spectra were collected over the range 190-250 nm, using a Jasco-815 spectropolarimeter (JASCO International Co.Ltd., Tokyo, Japan) coupled to a Neslab RTE111 circulating water bath. The wavelength was calibrated using d-10-camphorsulfonic acid. Spectra were obtained at $25^{\circ} \mathrm{C}$ using $0.2 \mathrm{~cm}$ path length cell, averaged over 10-30 scans, at a scan speed of $20 \mathrm{~nm} / \mathrm{min}$, bandwidth of $1.0 \mathrm{~nm}, 0.5 \mathrm{~s}$ response and $0.1 \mathrm{~nm}$ resolution. $\mathrm{CD}$ spectra were obtained at $10.0 \mu \mathrm{M}$ peptide concentration in different environments: Tris/H3BO3 buffer, trifluoroethanol (TFE)/water mixture $(30 \% \mathrm{v} / \mathrm{v})$, and, in the presence of SUVs, zwitterionic POPC and anionic POPC/POPG (80:20). Following baseline correction, the observed ellipticity, $\theta$ (mdeg), was converted to mean residue ellipticity $[\Theta]\left(\mathrm{deg} \mathrm{cm}^{2} / \mathrm{dmol}\right)$, using the relationship $[\Theta]=100 \theta /(l \times c \times n)$ where $l$ is the path length in centimeters, $c$ is the millimolar concentration and $n$ is the number of residues in the peptide. The observed mean residue ellipticity in $222 \mathrm{~nm}\left(\Theta_{222}^{\text {obs }}\right)$ was converted to $\alpha$-helix fraction $\left(f_{\mathrm{H}}\right)$ using the method proposed (Deber and Li 1995):

$f_{\mathrm{H}}=\frac{\Theta_{222}^{o b s}-\Theta_{222}^{0}}{\Theta_{222}^{100}-\Theta_{222}^{0}}$

$\Theta_{222}^{0}=0$ and $\Theta_{222}^{100}$ is given by:

$\Theta_{222}^{100}=-39500(1-2.57 / n)$

$n$ is peptide length, $n=18$ in the present study.

Conformational analysis by molecular dynamics (MD) simulations.

The MD simulations were performed in TFE/water $(\sim 30 \% \mathrm{v} / \mathrm{v})$ mixture. We assumed a perfect alpha-helix as initial conformation for all peptides, which were built from their amino acid sequence using Molefacture plugin of VMD (Humphrey et al. 1996). Cubic simulation boxes with $4.4 \mathrm{~nm}$ length were build containing 1 peptide molecule, 200 TFE molecules, 1890 water molecules and 2 or 3 chloride $\left(\mathrm{Cl}^{-}\right)$counterions, which neutralize the net charge of each peptide. For all peptides the $\mathrm{C}$-terminus was amidated. For L1A the $\mathrm{N}$-terminus was treated as a positively charged group $\left(\mathrm{NH}_{3}{ }^{+}\right)$, in Ac-L1A the N-terminus was acetylated and in Abz-L1A-W8V we attached an o-amino-benzoic acid (Anthranilic acid, or Abz) to N-terminus. The MD simulations were performed in GROMACS 4.5.6 (Pronk et al. 2013) package using GROMOS 96 (Schuler et al. 2001) force field. Simple Point Charge (SPC) model (Berendsen et al. 1981) was used for water while for the TFE the model proposed by Fioroni (Fioroni et al. 2000) was used. The ABZ molecule were modeled using Automated Topology Builder (ATB) website (Malde et al. 2011). The system minimizations was carried out in 8000 steps of conjugate gradient and a steepest descent cycle was performed every 500 steps. Molecular dynamics with position restraints applied to peptide backbone for solvent and ions relaxation ran for $1 \mathrm{~ns}$ with $2 \mathrm{fs}$ time-step. The constraints were then removed and the system underwent a $50 \mathrm{~ns}$ MD simulations. Periodic boundary conditions have been imposed in the system simulation with a cutoff radius of $1.4 \mathrm{~nm}$. The LINCS (Hess et al. 1997) algorithm was used to constraint all bond lengths and SETTLE (Miyamoto and Kollman 1992) to constraint water geometry. Simulations ran in the canonical ensemble (NTP) $300 \mathrm{~K}$ and $1 \mathrm{~atm}$. Temperature and pressure were modulated using Berendsen coupling algorithms (Berendsen et al. 1984) with coupling and isothermal compressibility constants of $0.01 \mathrm{ps}$ and $65 \mu \mathrm{Bar}^{-1}$ respectively. The cutoff radius for van der Waals interactions was $1.4 \mathrm{~nm}$ and long-range electrostatic interactions were treated using Particle Mesh Ewald (PME) method (Darden et al. 1993).

\section{Results}

\section{Antibacterial and hemolytic activities}

The antibacterial activities of L1A and its analogs were assessed estimating their minimum inhibitory concentrations (MIC). Table 1 shows also the MIC for the control, chloramphenicol, and includes MIC observed for the peptide Polybia- MP1, or shortly MP1, a non-hemolytic and potent broad spectrum antimicrobial peptide (de Souza et al. 2009). The strains tested with L1A and analogs are not the same tested with MP1 and the table includes also the ratios of the MICs of MP1 and chloramphenicol. L1A and analogs were more efficient against the two Gram negative bacteria strains investigated and displayed lower efficacy against $S$. aureus. Considering the Gram-negative bacteria, however, the comparison of the ratios between the MICs of peptides and those of the control for L1A and for MP1 shows that the efficiency of L1A is similar to MP1. The decreased efficacy observed for the analogs is probably related to their lower net charge that could result in weaker electrostatic interaction with the anionic 
Table 1 Biological activities and hemolysis of synthetic peptides

\begin{tabular}{|c|c|c|c|c|}
\hline \multirow[t]{2}{*}{ Peptides $(\mu \mathrm{M})$} & \multicolumn{2}{|l|}{ Gram- } & \multirow{2}{*}{$\begin{array}{l}\text { Gram+ } \\
\text { S. aureus }\end{array}$} & \multirow[t]{2}{*}{ Hemolysis $(\%)^{\mathrm{a}}$} \\
\hline & E. coli & P. aeruginosa & & \\
\hline IDGLKAIWKKVADLLKNT-NH ${ }_{2}$ & 4.6 & 37.0 & $>74.0$ & 4.0 \\
\hline Ac-IDGLKAIWKKVADLLKNT-NH ${ }_{2}$ & 9.0 & 72.5 & $>72.5$ & 5.0 \\
\hline Abz-IDGLKAIVKKVADLLKNT-NH ${ }_{2}$ & 9.0 & 70.0 & $>70.0$ & 46.0 \\
\hline Chloramphenicol & 7.7 & $>124.0$ & 30.9 & \\
\hline Polybia-MP1/chloroamphenicol & $5.0 / 12.0$ & $5.0 / 22.0$ & $9.0 / 22.0$ & \\
\hline Polybia-MP1 & - & - & - & 5 \\
\hline Melittin & & & & 100 \\
\hline
\end{tabular}

${ }^{a}$ Percentage of hemolysis at a $300 \mu \mathrm{g}$ of the peptides or 148, 145, 140 and $110 \mu \mathrm{M}$ of the L1A, Ac-L1A, Abz-L1A-W8V and melittin. Chloramphenicol and melittin were used as controls for antibacterial and hemolytic activities respectively lipopolysaccharides of the outer membrane of Gram negative bacteria. The hemolytic activity was evaluated at a peptide concentration which is twice the largest concentration used to kill bacteria. It was observed only 4-5\% of hemolysis for L1A and the acetylated analog, which are very low compared to the almost $100 \%$ for mellitin used as control. This low hemolytic activity is comparable to those of MP-1 (Souza et al. 2005). The Abz analog induced, however, surprisingly around ten times the hemolysis observed for L1A and Ac-L1A. The N-terminus acetylation lead the analog with the same efficiency of the parent peptide, however, the covalently labeling of the
N-terminus with Abz impaired the antibacterial efficiency and imparted undesirable hemolytic activity. Aiming to understand the effect of these modifications we explored the lytic activity of the peptide and analogs in model membranes that are more simple systems.

\section{Lytic activity in liposomes}

The membrane permeation activities of the peptide L1A and its analogs Ac-L1A and Abz-L1A-W8V were investigated by dye leakage experiments. In these experiments aliquots of vesicles containing entrapped fluorescent dye
Fig. 1 Dose response curves for L1A and analogs induced leakage of carboxyfluorescein entrapped LUVs. The percentage of leakage is shown as a function of the ratio of peptide to total lipid molar concentrations after $1 \mathrm{~min}$ of peptides action in A- POPC and BPOPC/POPG (8:2) LUVs for L1A (squares), Ac-L1A (up triangles) and Abz-L1A-W8V (circles). Total lipid concentration $100 \mu \mathrm{M}$

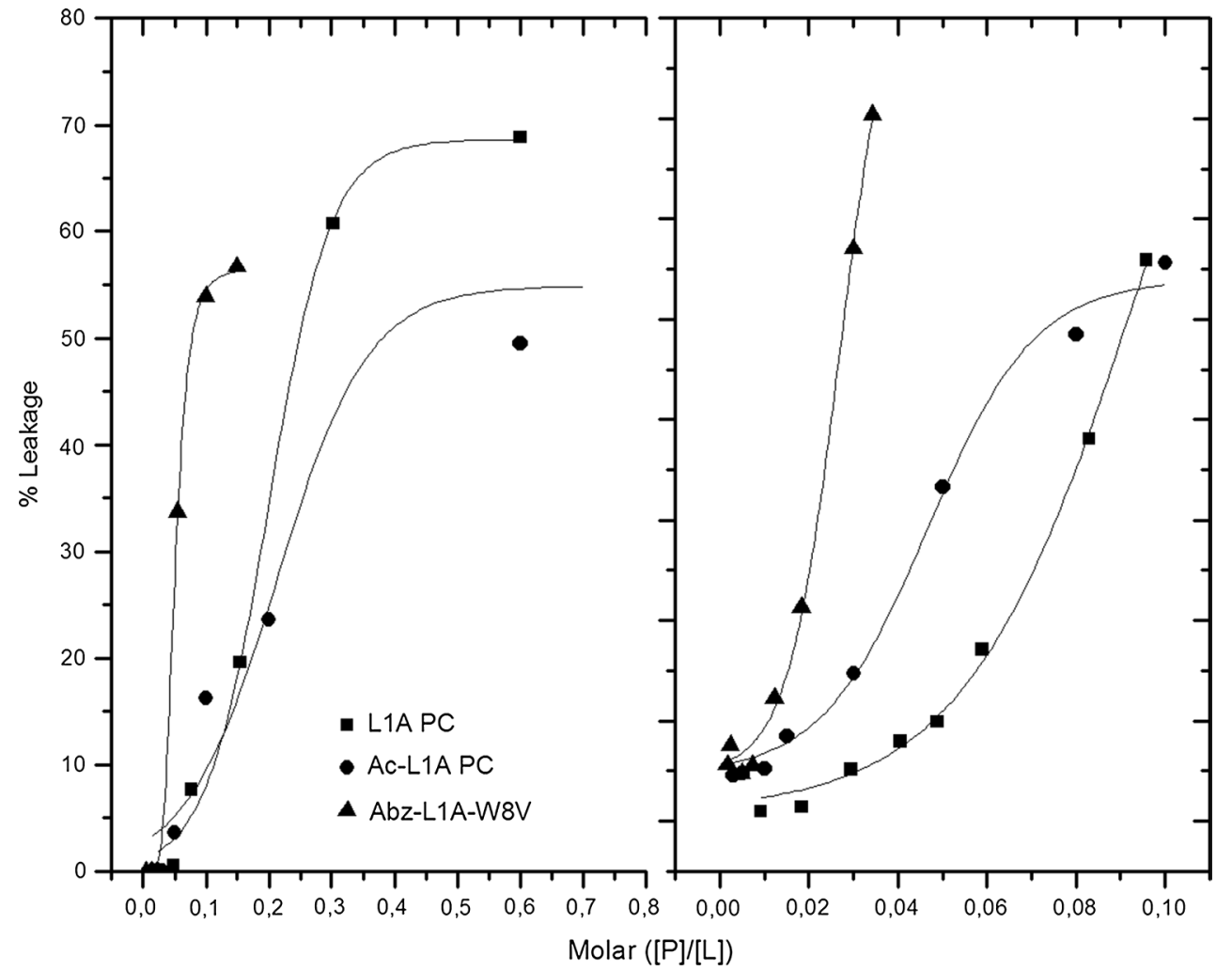




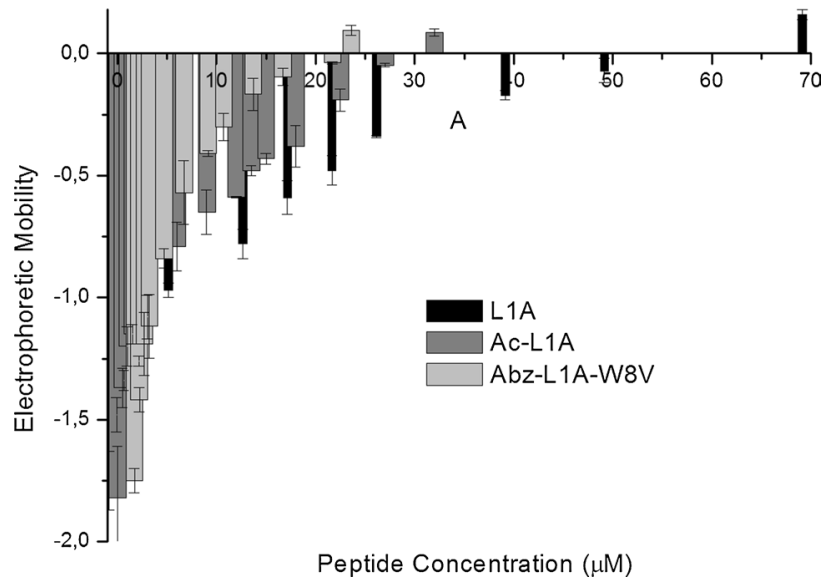

Fig. 2 Electrophoretic mobility of POPC/POPG (8:2) large unilamellar vesicles (110 $\mathrm{nm}$ diameter) as a function of total peptide concentration when titrated with aliquots of L1A (triangles), Ac-L1A (circles) and Abz-L1A-W8V (squares) at $40 \mu \mathrm{M}$ total lipid concentration in Tris buffer $10-150 \mathrm{mM} \mathrm{NaCl}$. The voltage applied in these experiments was around $50.0 \pm 2.0 \mathrm{~V}$

carboxyfluoresceine are added to a peptide solution and the de-quenching of the dye was monitored as a function of the elapsed time. The addition of vesicles was followed by an exponential growth in the fluorescence intensity; such that, after just 1 min the leakage was almost complete. The dose-response curves, percentage of leaked dye (\% leakage) vs the peptide to lipid molar concentration ratios ([P]/ [L]), obtained after $1 \mathrm{~min}$ of vesicle addition are shown in Fig. 1. The $[\mathrm{P}] /[\mathrm{L}]$ ratios to achieve $50 \%$ of dye release $\left([\mathrm{P}] /[\mathrm{L}]_{50}\right)$ indicate that these peptides are more efficient in anionic vesicle. The $[\mathrm{P}] /[\mathrm{L}]_{50}$ values obtained in zwitterionic vesicle after $1 \mathrm{~min}$ of peptide action for L1A, AcL1A and Abz-L1A are 0.24, 0.37 and 0.06 and in anionic vesicles $0.09,0.075$ and 0.027 respectively. Although these results emphasize that the vesicles charge is important to the peptide selectivity the $([\mathrm{P}] /[\mathrm{L}])_{50}$ values revealed that the analogs, with lower net charges, were more efficient to permeate vesicles except Ac-L1A in POPC vesicles. The lytic activities observed in zwitterionic vesicle were well correlated with their hemolytic activities, however this correlation cannot be observed for the antibacterial activities. Looking for a comprehension of this unexpected charge effects on the lytic activity of these peptides in anionic vesicles, we have explored their affinities to these vesicles by measuring the changes in the electrophoretic mobility due to the peptide adsorption to the vesicles.

\section{Electrophoretic mobility titrations}

The peptide adsorption to POPC/POPG (80:20) vesicles was monitored by changes in the electrophoretic mobility. In these experiments POPC/POPG vesicles suspensions,

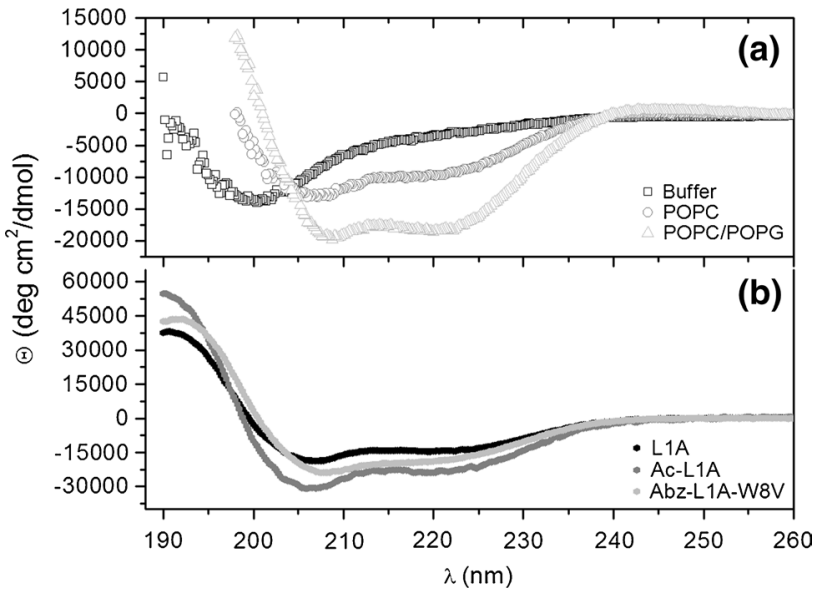

Fig. 3 a Circular dichroism spectra of Abz-L1A-W8V at $20 \mu \mathrm{M}$ in TRIS buffer in the absence and the presence of SUVs at $400 \mu \mathrm{M}$ of vesicles POPC and POPC/POPG (80:20). b Circular dichroism spectra of L1A, Ac-L1A and Abz-L1A-W8V in TFE $30 \%$ aqueous solution

at $40 \mu \mathrm{M}$ total lipid concentration, were titrated with peptides. Figure 2 shows the values of the electrophoretic mobility as a function of the peptide concentration added. In the absence of peptide, vesicle's mobility was around $-1.8 \mu \mathrm{m} \mathrm{cmV}^{-1} \mathrm{~s}^{-1}$ and with subsequent peptide additions the mobility becomes less negative decreased up to its inversion to positive values. Unexpectedly, the concentrations of the peptides to achieve the inversion of the mobility signal from negative to positive were lower for the analogs with lower net charges. This result suggests that the affinities of the analogs to anionic vesicle are higher in comparison to L1A in accordance with the higher lytic efficiency of the analogs in anionic vesicles. The higher affinities of the analogs to anionic vesicles as well as their lytic efficiencies in these vesicles could be due to a better amphipathic structure achieved by the peptide in these vesicles. Trying to check this working hypothesis we performed a conformational analysis of the L1A and analogs in an helicogenic solvent such as TFE and in lipid vesicles.

\section{Conformational analysis by CD}

The CD spectra of the peptides in water or buffer showed a negative band near $200 \mathrm{~nm}$ and only a slight drift from the base line in $220 \mathrm{~nm}$ indicating random coil conformation. In TFE $30 \%$ aqueous solution two negative dichroic bands, in 222 and $208 \mathrm{~nm}$, were observed in the CD spectra, that is a signature of helical conformation. The total helical fractions, determined from the spectra were $42 \pm 5 \%$ for L1A, $72 \pm 3 \%$ for the acetylated analog and $55 \pm 5 \%$ for Abz-analog. Helical conformation was also observed when the peptides were in contact with zwitterionic and anionic 
Table 2 Structural parameters and helix fractions in TFE $30 \%, \mathrm{POPC}$ and POPC/POPG (80:20) vesicles for L1A, Ac-L1A, Abz-L1A-W8V

\begin{tabular}{|c|c|c|c|c|c|c|c|}
\hline & \multicolumn{2}{|c|}{ L1A } & Ac-L1A & \multicolumn{4}{|c|}{ Abz-L1A-W8V } \\
\hline$Q$ & \multicolumn{2}{|l|}{+3} & +2 & \multicolumn{2}{|l|}{+2} & & \\
\hline$\langle\mathrm{H}\rangle$ & \multicolumn{2}{|c|}{-0.11} & -0.1 & \multicolumn{2}{|l|}{-0.1} & & \\
\hline$\mu$ & \multicolumn{2}{|c|}{0.36} & 0.36 & \multicolumn{2}{|l|}{0.36} & & \\
\hline$\Phi$ & \multicolumn{2}{|l|}{140} & 140 & \multicolumn{2}{|l|}{140} & & \\
\hline \multirow[t]{2}{*}{$f \alpha$ TFE $30 \%$} & \multicolumn{2}{|c|}{$0.42 \pm 0.05$} & $0.72 \pm 0.03$ & \multicolumn{2}{|c|}{0.550 .05} & & \\
\hline & & $\mathrm{PC}$ & $\mathrm{PC} / \mathrm{PG}^{\mathrm{a}}$ & $\mathrm{PC}$ & $\mathrm{PC} / \mathrm{PG}^{\mathrm{a}}$ & $\mathrm{PC}$ & $\mathrm{PC} / \mathrm{PG}^{\mathrm{a}}$ \\
\hline \multicolumn{2}{|l|}{$f_{\mathrm{h}}$ Vesicles } & 0.11 & 0.19 & $0.26^{\mathrm{a}}$ & $0.49^{\mathrm{a}}$ & $0.32^{\mathrm{a}}$ & $0.53^{\mathrm{a}}$ \\
\hline \multicolumn{2}{|l|}{$\Delta \lambda_{\max }$} & 28 & 32 & 34 & 35 & 6 & 6 \\
\hline \multicolumn{2}{|c|}{$\mathrm{K}_{\mathrm{SVV}} / \mathrm{K}_{\mathrm{SVB}}$} & 0.6 & 0.27 & 0.31 & 0.15 & 0.28 & 0.11 \\
\hline \multicolumn{2}{|c|}{$[\mathrm{P}] /[\mathrm{L}]$ at $\mathrm{EC}_{50}$ vesicles } & 0.08 & 0.04 & 0.2 & 0.03 & 0.03 & 0.02 \\
\hline
\end{tabular}

${ }^{\text {a }}$ Vesicles POPC/POPG (80:20) vesicles, as shown in the Fig. 3 for Abz-L1A-W8V. These spectra show that the $208 \mathrm{~nm}$ band is more intense than $222 \mathrm{~nm}$ suggesting that up to this lipid to peptide molar concentration ratio $([\mathrm{L}] /[\mathrm{P}]=20)$ the peptides are in the monomeric form in the vesicles (Lau et al. 1984). The helical fractions calculated from the CD spectra are shown in the Table 2. Their helical contents in anionic vesicles are roughly twice as those observed in zwitterionic vesicles. However, the helical fractions, in vesicles, even in anionic ones, are somewhat different from those measured in TFE. The helical fractions of the analogs in POPC/POPG vesicles, at $[\mathrm{L}] /[\mathrm{P}] \sim 20$, were more than twice compared to L1A indicating that the N-terminus modification contributed to optimize the helical structure of the these peptides in vesicles. The conformational analysis by $\mathrm{CD}$ was complemented with MD simulations aiming to explore the amphipathic structure.

\section{Molecular dynamics simulations}

The MD simulations showed that after 50 ns the peptides displayed significant helical content in TFE. Evaluating the hydrogen bonds between the $\mathrm{CO}$ groups of the backbone of a residue with the $\mathrm{NH}$ groups of its fourth neighbor residue $(i, i+4)$ simultaneously with the backbone dihedral angles $(\varphi, \psi)$ for the timeframes throughout the simulations, we observe that for L1A, in average, 7 hydrogen bonds (HB) are stable for more than $70 \%$ of the simulation time. Three of these HB G3-I7, L4-W8 and K5-K9, are stable for $80 \%$ of the overall time as shown in the Fig. 4. The residues involved in these HB contribute to the stabilization of the helical conformation resulting in a helical fraction around $40 \%$. The $\mathrm{N}$-acetylation resulted in five extra backbone hydrogen bonds among the residues D2-A6; A6-K10; I7-V11 and W8-A12 which stable for around $80-90 \%$ of the time and K9-D13 stable along $70 \%$ of the simulation time, resulting in a helical fraction around $70 \%$. For the

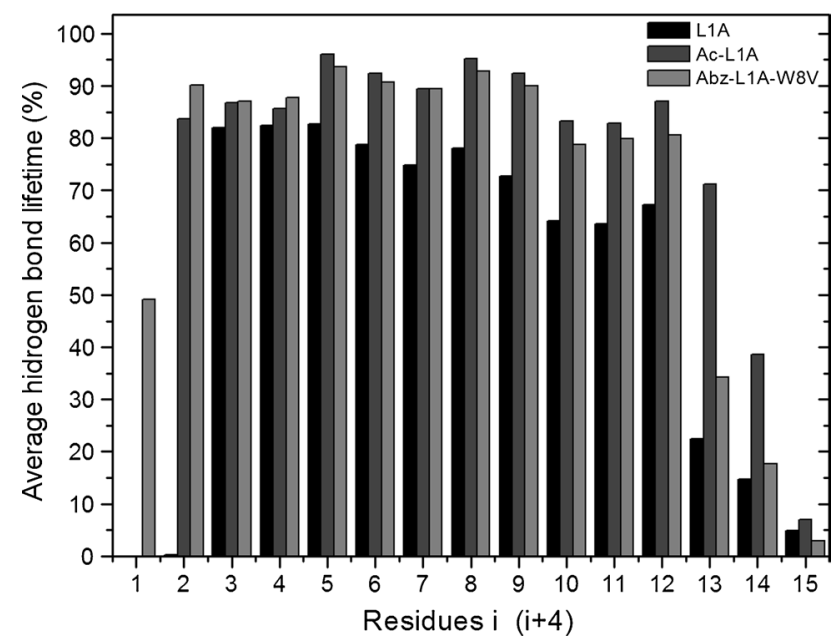

Fig. 4 Percentage average lifetime of hydrogen bonds between residues $i$ and $i+4$ of peptide backbone obtained from $50 \mathrm{~ns}$ molecular dynamics simulations

Abz- analog it was observed that the number of backbone HB is 11 , resulting in $60 \%$ of helix. Considering experimental errors, the helical content observed in our simulations are similar to the results from CD analysis in TFE. The simulations showed also that the $\mathrm{CO}$ group from Abz interacts with the $\mathrm{NH}$ of the backbone of Leu4 in the analog Abz-L1A-W8V.

The distribution of each kind of solvent around the peptide was performed by the analysis of the Radial Distribution Function (RDF) and Spatial Distribution Function (SDF). Figure 5 shows the RDF obtained from the center of mass of the tryptophan to the $\mathrm{CF} 3$ and $\mathrm{OH}$ of TFE and to the $\mathrm{OH}$ of water for L1A and Ac-L1A. These plots shows that the CF3 group of the TFE is equally in close contact with tryptophan of the two peptides while water is layered beyond the TFE layer. These analyses also showed that their hydrophobic faces were surrounded by TFE molecules 


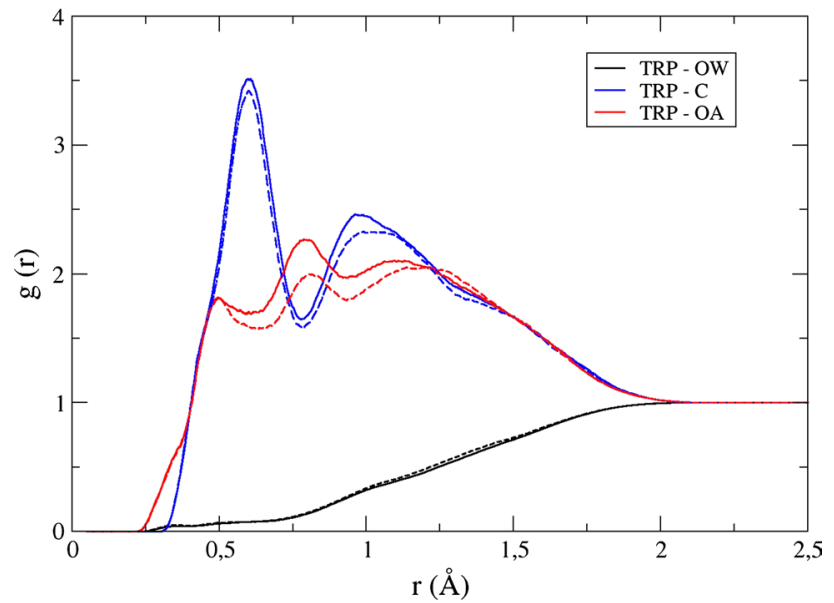

Fig. 5 Radial Distribution Function (RDF) between center of mass of TRP side chain and the oxygen atom of water (OW, in black), the carbon atom $\left(\mathrm{C}\right.$, in blue) of $\mathrm{CF}_{3}$ group and oxygen atom (OA, in red) of $\mathrm{OH}$ group of TFE for L1A (solidlines) and Ac-L1A (dashed lines) (color figure online)

while their hydrophilic faces were in contact with water molecules (Fig. 6). The amidated C-terminals of the three peptides are buried in TFE as an extension of their hydrophobic faces. Significant differences in the SDF were observed in the N-terminus region of each peptide. Water surrounds preferentially the L1A charged N-terminus. Otherwise, the uncharged $\mathrm{N}$-terminals of Ac-L1A and Abz-L1A-W8V are in close contact with TFE molecules. These differences explained why the L1A N-terminus loses the structure in the course of MD simulations, since the presence of water molecules near the backbone prevents the formation of intra-chain CO-NH H-bonds. This was not observed for the two analogs to which an extra H-bond involving $\mathrm{CO}$ of $\mathrm{I} 1$ and $\mathrm{NH}$ of $\mathrm{K} 5$ is formed as a possible consequence of the competition between water and TFE in solvating these groups. Interestingly the Abz-analog N-terminus is strongly surrounded by TFE molecules, consistent with both the blue shift and the screening of the Abz by solvent molecules observed from quenching experiments with acrylamide (Zanin et al. 2013). In this way, the L1A N-terminus is in the hydrophilic face while for the analogs they are in the hydrophobic faces.

The hydrophilic faces of the peptides, preferentially solvated by water molecules, are composed of acidic, basic and polar residues distributed in two charge clusters that could contribute to stabilize the amphipathic helical structure, through hydrogen bonding or saline bridges among lateral chains. Aiming to identify whether saline bridges or side chain H-bonds are responsible for the stabilization of the helical structure or not, the average distances between opposed charged groups and between Asp13 and Asn17 were calculated from the MD simulations. These distances are displayed in the Table 3.
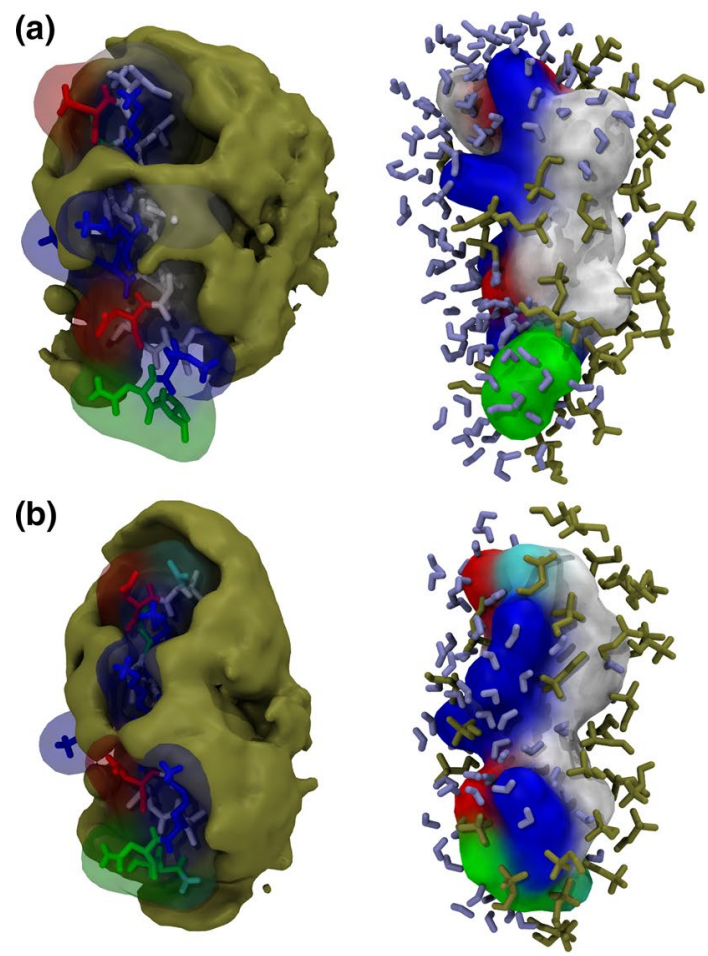

(c)
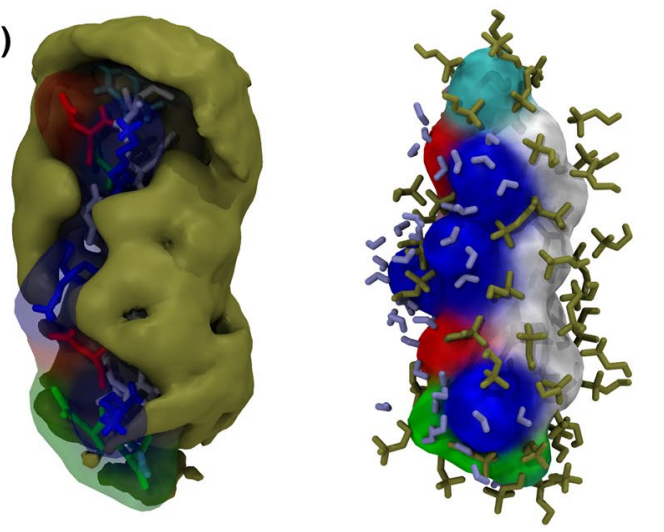

Fig. 6 Distribution of solvent molecules around each peptide. a L1A. b Ac-L1A. c Abz-L1A-W8V. Left spatial distribution function (SDF) of TFE molecules at density isovalue of $1.37 \mathrm{AU} / \AA^{3}$. Water distribution function is not shown for clarity. Right typical configuration of water and TFE molecules according SDF results. TFE is represented as surfaces or molecules in gold, the peptides in licorice and as transparent or opaque surfaces and water molecules as licorice in ice blue. The hydrophobic amino acids are colored as white, polar as green, negatively charged as red, positively charged as blue and neutral $\mathrm{N}$-terminals as cyan (color figure online)

These two groups of charges are centered in the aspartic acid residues. One with the aspartic acid D2 interacting with the $\mathrm{N}$-terminus and the neighboring lysine (K5) and a second group formed by the aspartic acid D13, the neighboring lysines (K9,K10 and $\mathrm{K} 16)$ and the asparagine N17. The distances between opposed charged residues in these groups are less than $10 \AA$. Judging from the distances obtained, only four residues would have a probabilistic 
Table 3 Average distance and standard deviation in Å between charged groups

\begin{tabular}{|c|c|c|c|c|c|c|}
\hline \multirow[t]{2}{*}{ Charged groups } & \multicolumn{2}{|l|}{ L1A } & \multicolumn{2}{|l|}{ Ac-L1A } & \multicolumn{2}{|l|}{ Abz-L1A-W8V } \\
\hline & Average distance & Standard deviation & Average distance & Standard deviation & Average distance & Standard deviation \\
\hline ASP2: $\mathrm{NH}_{3}-$ Terminus & 7.2 & 0.7 & - & - & - & - \\
\hline ASP2:LYS5 & 5.5 & 1.4 & 6.1 & 1.9 & 5.4 & 1.5 \\
\hline ASP2:LYS9 & 11.9 & 1.7 & 10.9 & 2.4 & 11.1 & 2.1 \\
\hline ASP2:LYS10 & 13.9 & 2.0 & 14.9 & 2.0 & 15.1 & 1.9 \\
\hline ASP2:LYS16 & 20.4 & 2.0 & 21.3 & 2.2 & 21.4 & 1.7 \\
\hline ASP13: $\mathrm{NH}_{3}-$ Terminus & 17.8 & 1.5 & - & - & - & - \\
\hline ASP13:LYS5 & 16.2 & 2.1 & 16.5 & 1.8 & 16.7 & 1.7 \\
\hline ASP13:LYS9: & 7.5 & 2.5 & 9.0 & 2.3 & 8.7 & 2.4 \\
\hline ASP13:LYS10 & 9.6 & 2.9 & 8.6 & 2.5 & 8.6 & 2.6 \\
\hline ASP13:LYS16 & 6.2 & 2.0 & 6.8 & 2.9 & 5.7 & 1.9 \\
\hline ASP13:ASN17 & 11.5 & 4.1 & 6.8 & 3.0 & 11.0 & 4.1 \\
\hline
\end{tabular}

The selected groups for this calculation are: $\mathrm{NH}_{3}$ side chains of lysine, $\mathrm{NH}_{3}$ terminal connected to Ile1 and $\mathrm{O}$ side chains of aspartic acid

chance to ion pairing D2 and K5 and D13 and K16, despite these distances are larger than $5 \AA$ when it should be expected to observe ion pairing at $4 \AA$ (Marqusee and Baldwin 1987). One can observe in this table that the standard deviations obtained for these distances are considerably large, suggesting great fluctuations in these distances. Trying to understand the origin of these fluctuations we displayed the distances data as histograms of the frequency of occurrence during simulations vs the distances from the center of mass of the $\mathrm{D} 13 \mathrm{COO}^{-}$group to those of the $\mathrm{NH}_{3}{ }^{+}$of lysines (K10 and K16) or of the $\mathrm{NH}_{2}$ of asparagine (N17). The histograms obtained for the three peptides are shown in the Fig. 7. One can observe in these histograms high frequency values for short and long distances that explain the large values of the average distances of the Table 3 as well as the large standard deviation.

The histogram of frequency of occurrence of ion pair vs distance for L1A evidences that short lived ion pairing is formed between D13 and K16 and in lesser extension between D13 and K9 whose highest occurrence near 5.5 $\AA$. For the acetylated analog however, the histogram shows two ion pairing D13- K16 and D13-N17 whose highest occurrences are centered around $4 \AA$. These two ion pairing are roughly equally favorable, and most likely would contribute to the higher stabilization of the helical structure in the C-terminus region of this analog. The histogram for the Abz-analog shows that D13-K16 ion pair is most frequent at a distance close to $6 \AA$ and in lesser extension it is accompanied by D13-K10 at the same distance. These histograms evidence that for the parent peptide L1A and the Abz-analog the salt bridges stabilizing the C-terminus region is less likely to occur, that is the source of the observed short live backbone ( $\mathrm{CO}-\mathrm{NH})$ hydrogen bonds and the consequent low helical stability of this region.

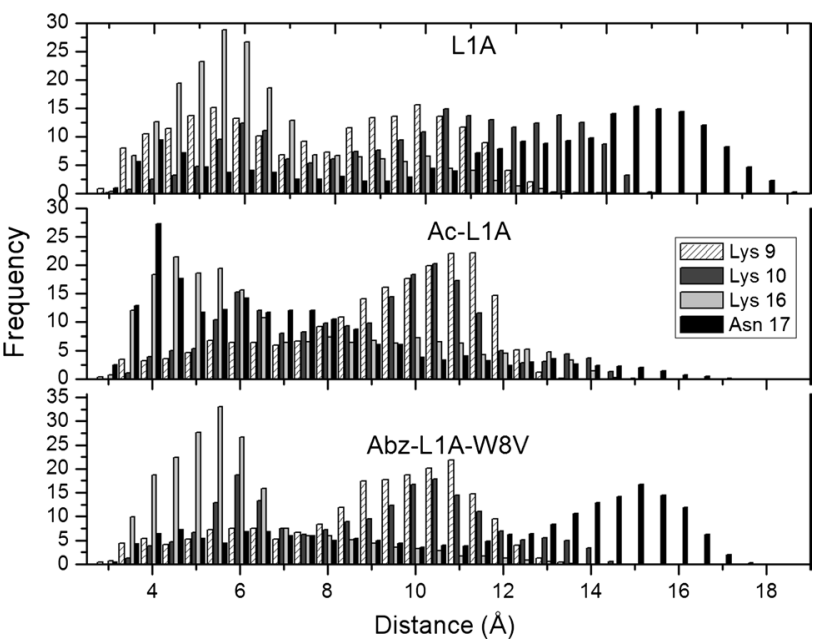

Fig. 7 Histogram analysis of distances between side chains of ASP13 and positive charged (LYS) or polar (ASN) residues near it for the three investigated peptides from MD simulations

These data seems to evidence that the state of the N-terminal has influenced the possibility of different ion-pairing in the C-terminus.

\section{Discussion}

A large body of evidences, raised in the last decades, indicates that the antimicrobial peptides act on bacterial membranes without the requisite of membrane receptors. Due to this fact, these peptides have a great potential to substitute conventional antibiotics, because acting only on the lipidic phase of the membrane would restrict the development of resistance mechanism by the bacteria. 
However, recently it was observed that some strains secrete proteases as an alternative resistance mechanism against antimicrobial peptides (Strömstedt et al. 2009; Guilhelmelli et al. 2013). Blocking both N- and C-terminals of the peptides, by chemical modifications, is a possible strategy to resists against proteolytic digestion (Andreu and Rivas 1998). Otherwise, these modifications have been shown to enhance the helical stability by decreasing the repulsive interaction of the $\alpha$-amino and the carboxyl groups with the positive and negative poles of the helical dipole (Fairman et al. 1989). Evidences have also shown that lateral chains can hydrogen bonding with unsatisfied main chain $\mathrm{NH}$ at the $\mathrm{N}$-terminus (Richardson and Richardson 1988) increasing the helical stability. The helical stability of peptides in physiological salt concentration and the consequent maintenance of their lytic and bactericidal activities is one of the challenges in developing new antibiotic compounds (Bahar and Ren 2013).

The conformational analysis by $\mathrm{CD}$ and MD simulations in TFE showed that the $\mathrm{N}$-terminus modifications resulted in the increase in the helical content. The effects of these end modifications associated with the acidic residue in the second position at $\mathrm{N}$-terminus contribute to the reduction of the repulsive interaction between $\mathrm{N}$ terminus and the helix macrodipole (Marqusee and Baldwin 1987). As a consequence the helical content of the analogs are higher compared to L1A. It is noteworthy that in the Abz-analog the tryptophan was substituted by valine that has slightly lower helix propensity than tryptophan (Zanin et al. 2013), however, Abz-L1A-W8V was less structured than the acetylated analog in $30 \%$ TFE as observed from the CD data. MD simulations showed that the $\mathrm{N}$-terminus modifications enhanced the backbone hydrogen bonds near the N-terminus for more than $70 \%$ of the simulation timeframes. The simulations also showed that the $\mathrm{N}$-termini of the analogs are in close contact with $\mathrm{CF} 3$ groups of TFE that means in less polar environment. MD simulations evidenced that the mechanism by which the N-modified analogs stabilizes the helical conformation is by extra hydrogen bonding and, in the case of $\mathrm{N}$-acetylated analog, by extra ion pairing by D13-K16 and D13-N17.

The comparison based on structure-function of the peptide L1A with its $\mathrm{N}$-terminal modified analogs has shown that the $\mathrm{N}$-terminal substitutions enhanced the affinity as well as their helical stability in lipid vesicles. Molecular dynamics simulations in TFE also showed that the $\mathrm{N}$-terminus region of the two analogs is more stable compared with L1A. The elimination of the amino group charge most likely reduces the repulsive interactions with the membrane dipole (da Silva et al. 2014) allowing more deep penetration of the peptide into the bilayer (Zanin et al. 2013) disturbing, more efficiently, the lipid packing of the bilayer, enhancing the lytic activities in vesicles. Despite improving the lytic activity in anionic vesicles, the $\mathrm{N}$-terminus modifications impaired the bactericide activity that could be due to the reduction of the peptide net charge. In spite of this, the analogs showed the same efficiency of chloramphenicol, used as antibiotic reference in inhibit growth of Gram negative bacteria and higher efficiency against $\mathrm{S}$. aureus than this antibiotic. The reduction of the peptide net charge due to the $\mathrm{N}$-terminus acetylation had no effect on the hemolytic activity. The increase in the hemolytic potency observed for the Abz analog is similar to that observed for the NBD labeled melittin (Raghuraman and Chattopadhyay 2007). They observed increased hemolytic activity for the NBD covalently bonded at the $\mathrm{N}$-terminus of melittin compared to the native peptide.

In conclusion, the L1A N-terminus acetylation results in the increase of the helical content in aqueous solution of TFE and in phospholipid anionic mixed vesicles, while the covalently attachment of 2-aminobenzoic acid at the amino group did not result in the same augment of helicity. Molecular dynamics simulations confirmed these experimental observations and showed that the hydrophobic face and the $\mathrm{N}$-terminus of the analogs are in close contact with TFE molecules and that this region is more helical structured than the parent peptide. The analysis of the pair distribution functions of the center of mass of the ionizable groups brought evidences that the modifications of the $\mathrm{N}$-terminus can influence ion-pairing in the acetylated analog favoring an extra backbone hydrogen bonding compared to the Abz-analog. The higher helical and, consequently, more optimized amphipathic structure allowed that the analogs have higher affinity to anionic vesicles that was well correlated with the lytic efficiency in these vesicles. The loss of the amino group charge lead to impairment of the antibacterial activity while the attachment of the Abz group to amino terminal resulted in undesirable hemolysis.

Acknowledments ASA and JRN acknowledge the financial support from São Paulo Research Foundation-FAPESP Grant \#2010/18169-3 and Grant \#2011/11640-5 respectively. ASA acknowledges the resources supplied by the Center for Scientific Computing (NCC/GridUNESP) of the São Paulo State University (UNESP) and CENAPAD-SP (Centro Nacional de Processamento de Alto Desempenho em São Paulo). JRN is researcher of CNPq. LPMZ was recipient of a PhD scholarship from CAPES.

\section{Compliance with ethical standards}

Conflict of interest The authors declare that they have no conflict of interest.

Ethical approval The human blood used in the hemolytic activity experiments were provided by volunteer donors after consultation of the Institutional Ethical Committee. 


\section{References}

Andreu D, Rivas L (1998) Animal antimicrobial peptides: an overview. Biopolymers 47:415-433. doi:10.1002/ (SICI)1097-0282(1998)47:6<415:AID-BIP2>3.0.CO;2-D

Bahar AA, Ren D (2013) Antimicrobial Peptides. Pharmaceuticals 6:1543-1575. doi:10.3390/ph6121543

Berendsen HJC, Postma JPM, van Gunsteren WF, Hermans J (1981) Interaction Models for Water in Relation to Protein Hydration. In: Pullman B (ed) Intermolecular Forces. Springer, Netherlands, pp 331-342

Berendsen HJC, Postma JPM, van Gunsteren WF et al (1984) Molecular dynamics with coupling to an external bath. J Chem Phys 81:3684-3690. doi:10.1063/1.448118

da Silva AVR, De Souza BM, Dos Santos Cabrera MP et al (2014) The effects of the C-terminal amidation of mastoparans on their biological actions and interactions with membrane-mimetic systems. Biochim Biophys Acta 1838:2357-2368. doi:10.1016/j. bbamem.2014.06.012

Darden T, York D, Pedersen L (1993) Particle mesh Ewald: an $\mathrm{N} \cdot \log (\mathrm{N})$ method for Ewald sums in large systems. J Chem Phys 98:10089-10092. doi:10.1063/1.464397

de Souza BM, Cabrera MPDS, Gomes PC et al (2015) Structureactivity relationship of mastoparan analogs: effects of the number and positioning of Lys residues on secondary structure, interaction with membrane-mimetic systems and biological activity. Peptides. doi:10.1016/j.peptides.2015.04.021

Deber CM, Li SC (1995) Peptides in membranes: helicity and hydrophobicity. Biopolymers 37:295-318. doi:10.1002/bip.360370503

de Souza BM, Silva AR, Resende VMF, Arcuri HA, dos Santos Cabrera MP, Ruggiero Neto J, Palma MS (2009) Characterization of two novel polyfunctional mastoparan peptides from the venom of the social wasp Polybia pulista. Peptides 30:1387-1395

Fairman R, Shoemaker KR, York EJ et al (1989) Further studies of the helix dipole model: effects of a free alpha-NH3+ or alphaCOO- group on helix stability. Proteins 5:1-7. doi:10.1002/ prot.340050102

Fioroni M, Burger K, Mark AE, Roccatano D (2000) A new 2,2,2-trifluoroethanol model for molecular dynamics simulations. J Phys Chem B 104:12347-12354. doi:10.1021/jp002115v

Fjell CD, Hancock REW, Cherkasov A (2007) AMPer: a database and an automated discovery tool for antimicrobial peptides. Bioinforma Oxf Engl 23:1148-1155. doi:10.1093/bioinformatics/ btm068

Guilhelmelli F, Vilela N, Albuquerque P et al (2013) Antibiotic development challenges: the various mechanisms of action of antimicrobial peptides and of bacterial resistance. Front Microbiol. doi:10.3389/fmicb.2013.00353

Hamill P, Brown K, Jenssen H, Hancock REW (2008) Novel antiinfectives: is host defence the answer? Curr Opin Biotechnol 19:628-636. doi:10.1016/j.copbio.2008.10.006

Hess B, Bekker H, Berendsen HJC, Fraaije JGEM (1997) LINCS: A linear constraint solver for molecular simulations. J Comput Chem 18:1463-1472. doi:10.1002/ (SICI)1096-987X(199709)18:12<1463:AID-JCC4>3.0.CO;2-H

Humphrey W, Dalke A, Schulten K (1996) VMD: visual molecular dynamics. J Mol Graph 14:33

Korkmaz B, Attucci S, Juliano MA, Kalupov T, Jourdan ML, Juliano L et al (2008) Measuring elastase, proteinase 3 and cathepsin G activities at the surface of human neutrophils with fluorescence resonance energy transfer substrates. Nat Protoc 3(6):991-1000

Lau SY, Taneja AK, Hodges RS (1984) Synthesis of a model protein of defined secondary and quaternary structure. Effect of chain length on the stabilization and formation of two-stranded alphahelical coiled-coils. J Biol Chem 259:13253-13261
Leite NB, da Costa LC, Dos Santos Alvares D et al (2011) The effect of acidic residues and amphipathicity on the lytic activities of mastoparan peptides studied by fluorescence and CD spectroscopy. Amino Acids 40:91-100. doi:10.1007/s00726-010-0511-9

Malde AK, Zuo L, Breeze M et al (2011) An Automated Force Field Topology Builder (ATB) and Repository: Version 1.0. J Chem Theory Comput 7:4026-4037. doi:10.1021/ct200196m

Marqusee S, Baldwin RL (1987) Helix stabilization by Glu-...Lys+ salt bridges in short peptides of de novo design. Proc Natl Acad Sci USA 84:8898-8902

Meletiadis J, Meis JF, Mouton JW et al (2000) Comparison of NCCLS and 3-(4,5-dimethyl-2-Thiazyl)-2, 5-diphenyl-2H-tetrazolium bromide (MTT) methods of in vitro susceptibility testing of filamentous fungi and development of a new simplified method. J Clin Microbiol 38:2949-2954

Miyamoto S, Kollman PA (1992) Settle: an analytical version of the SHAKE and RATTLE algorithm for rigid water models. J Comput Chem 13:952-962. doi:10.1002/jcc.540130805

Pronk S, Páll S, Schulz R et al (2013) GROMACS 4.5: a high-throughput and highly parallel open source molecular simulation toolkit. Bioinformatics 29:845-854. doi:10.1093/bioinformatics/btt055

Pushpanathan M, Gunasekaran P, Rajendhran J (2013) Antimicrobial peptides: versatile biological properties. Int $\mathrm{J}$ Pept 2013:e675391. doi:10.1155/2013/675391

Raghuraman H, Chattopadhyay A (2007) Orientation and dynamics of melittin in membranes of varying composition utilizing NBD fluorescence. Biophys J 92:1271-1283. doi:10.1529/ biophysj.106.088690

Richardson JS, Richardson DC (1988) Amino acid preferences for specific locations at the ends of alpha helices. Science 240:1648-1652

Rouser G, Fleischer S, Yamamoto A (1970) Two dimensional thin layer chromatographic separation of polar lipids and determination of phospholipids by phosphorous analysis of spots. Lipids. 5:494-496

Santos Cabrera dos MP, Costa STB, de Souza BM et al (2008) Selectivity in the mechanism of action of antimicrobial mastoparan peptide Polybia-MP1. Eur Biophys J EBJ 37:879-891. doi:10.1007/s00249-008-0299-7

Schuler L, Daura X, Van Gunsteren W (2001) An improved GROMOS 96 force field for aliphatic hydrocarbons in the condensed phase. J Comput Chem 22:1205-1218

Souza BM, Mendes MA, Santos LD et al (2005) Structural and functional characterization of two novel peptide toxins isolated from the venom of the social wasp Polybia paulista. Peptides 26:2157-2164. doi:10.1016/j.peptides.2005.04.026

Souza BM, dos Santos Cabrera MP, Gomes PC, Dias NB, Stabeli RG, Leite NB, Ruggiero Neto J, Palma MS (2015) Structure-activity relationship of mastoparan analogs: Effects of the number and positioning of Lys residues on secondary structure, interaction with membrane mimetic systems and biological action. Peptides 72:164-174

Strömstedt AA, Pasupuleti M, Schmidtchen A, Malmsten M (2009) Evaluation of Strategies for Improving Proteolytic Resistance of Antimicrobial Peptides by Using Variants of EFK17, an Internal Segment of LL-37. Antimicrob Agents Chemother 53:593-602. doi:10.1128/AAC.00477-08

Teixeira V, Feio MJ, Bastos M (2012) Role of lipids in the interaction of antimicrobial peptides with membranes. Prog Lipid Res 51:149-177. doi:10.1016/j.plipres.2011.12.005

Wang Z, Wang G (2004) APD: the antimicrobial peptide database. Nucleic Acids Res 32:D590-D592. doi:10.1093/nar/gkh025

Wang G, Watson KM, Peterkofsky A, Buckheit RW (2010) Identification of novel human immunodeficiency virus type 1-inhibitory peptides based on the antimicrobial peptide database. Antimicrob Agents Chemother 54:1343-1346. doi:10.1128/AAC.01448-09 
Zanin LMP, dos Alvares DS, Juliano MA et al (2013) Interaction of a synthetic antimicrobial peptide with model membrane by fluorescence spectroscopy. Eur Biophys J 42:819-831. doi:10.1007/ s00249-013-0930-0
Zasloff M (2002) Antimicrobial peptides of multicellular organisms. Nature 415:389-395. doi:10.1038/415389a 